\title{
Reconfigurable Multirate Systems in Cognitive Radios
}

\author{
Amir Eghbali and Håkan Johansson \\ Division of Electronics Systems, Department of Electrical Engineering, \\ Linköping University \\ Sweden
}

\section{Introduction}

One perspective in communication systems is to increase the spectrum utilization using cognitive radios. A cognitive radio is a network of intelligent co-existing radios which senses the environment to find the available frequency slots, white spaces, or the spectrum holes as noted in Akyildiz et al. (2008); Haykin (2005). Then, it modifies its transmission characteristics to use that particular frequency slot. Figure 1 illustrates the overlay spectrum sharing outlined in Cabric et al. (2006), or the opportunistic spectrum access discussed in Zhao \& Sadler (2007); Zhao \& Swami (2007), or the dynamic spectrum access which is considered in Sherman et al. (2008). Here, secondary users occupy the frequency slots which are not used ${ }^{1}$ by the primary users. One of the main tasks in a cognitive radio is thus the spectrum mobility as in Akyildiz et al. (2006; 2008), or the dynamic frequency allocation as in Haykin (2005), or the dynamic spectrum allocation as in Leaves et al. (2004); Zhao \& Swami (2007). This chapter uses the term dynamic frequency-band allocation (DFBA). Being dynamic means that the transmission parameters, e.g., bandwidth, center frequency, transmission power, and communication standard etc., may vary with time according to Akyildiz et al. (2006). One should at least be able to change the center frequency and bandwidth although other parameters may also change. This is also referred to as the reconfigurability according to Akyildiz et al. (2008); Haykin (2005); Jondral (2005); Leaves et al. (2004); Ramacher (2007); Sherman et al. (2008).

Another perspective in communication systems calls for satellites to play a complementary role in supporting various wideband services as proposed by Arbesser-Rastburg et al. (2002); Evans et al. (2005); Farserotu \& Prasad (2000); Lucente et al. (2008); Nguyen et al. (2002); Re \& Pierucci (2002); Wittig (2000). For this purpose, the European space agency has proposed three major network structures for broadband satellite-based communication systems as in Arbesser-Rastburg et al. (2002). This requires an efficient use of the limited available frequency spectrum by satellite on-board signal processing as discussed in Abdulazim \& Göckler (2005a;b; 2006); Abdulazim et al. (2007); Arbesser-Rastburg et al. (2002); Eghbali et al. (2007a;b; 2009a); Eghbali, Johansson, Löwenborg \& Göckler (2011); Evans et al. (2005); Farserotu \& Prasad (2000); Göckler \& Abdulazim (2005; 2007);

\footnotetext{
${ }^{1}$ Under certain conditions, the secondary users need not wait for a vacant channel. This allows a simultaneous transmission over the same time or frequency as noted in Devroye et al. (2006).
} 


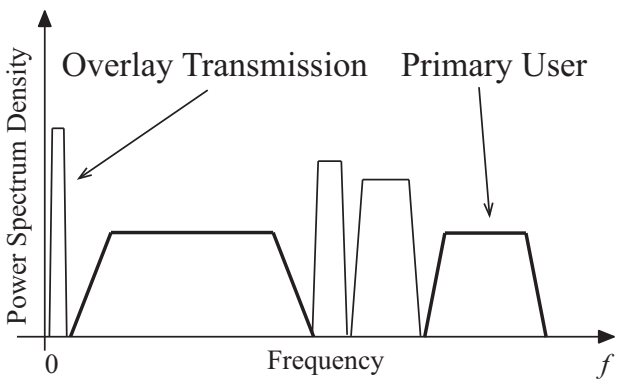

Fig. 1. Overlay approach for spectrum sharing.

Johansson \& Löwenborg (2005; 2007); Lippolis et al. (2004); Lucente et al. (2008); Nguyen et al. (2002); Re \& Pierucci (2002); Rosenbaum et al. (2006); Wittig (2000).

Like satellite-based communication systems which require both on-ground DFBA and on-board dynamic frequency-band reallocation (DFBR), the ad hoc- or infrastructure-based cognitive radios can also utilize DFBA and DFBR. In the ad hoc-based networks, individual users can utilize DFBA while DFBR can be performed by the base stations of infrastructure-based networks. The DFBA can also be deployed by the individual users of infrastructure-based networks. Both DFBA and DFBR need interpolation/decimation with variable parameters. For large sets of variable conversion factors, the implementation complexity increases. Complexity reduction can be achieved using reconfigurable structures which perform various tasks by simple modifications and without hardware changes. Also, the filter coefficients do not change thereby enabling us to solve the filter design problem only once and offline. Specifically, one must be able to reprogram the same hardware.

This chapter discusses the structure, reconfiguration, and the parameter selection when adopting the DFBA and DFBR for cognitive radios. Two approaches, i.e., Approach I and II, are discussed. They are appropriate based on the availability of (i) a composite signal comprising several user signals, or (ii) the individual user signals. Combinations of Approaches I and II provide increased freedom to allocate and reallocate the user signals.

\section{Basics of multirate signal processing}

This section treats some basics of sampling rate conversion (SRC), filter banks (FBs), perfect reconstruction (PR), and transmultiplexers (TMUXs).

\subsection{Sampling rate conversion: conventional}

Different parts of a multirate system operate at different sampling frequencies thereby necessitating interpolation (decimation) to increase (decrease) the sampling frequency of digital signals as outlined in Mitra (2006); Vaidyanathan (1993). Interpolation and decimation comprise lowpass filters as well as upsamplers and downsamplers whose block diagrams are shown in Fig. 2. In Fig. 2(a),

$$
y(n)=x(n M) \Longleftrightarrow Y(z)=\frac{1}{M} \sum_{k=0}^{M-1} X\left(z^{\frac{1}{M}} W_{M}^{k}\right), \quad W_{M}=e^{-j \frac{2 \pi}{M}}
$$


(a)

$$
\begin{aligned}
& x(m) \rightarrow \square \\
& M \rightarrow y(n)
\end{aligned}
$$

Fig. 3. Decimation by $M$.

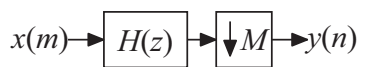

(b)

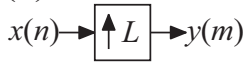

Fig. 2. (a) $M$-fold downsampler. (b) $L$-fold upsampler.

Note that $X\left(z^{\frac{1}{M}}\right)$ is not periodic by $2 \pi$ but adding the shifted versions gives a signal $Y(z)$ with a period of $2 \pi$ such that the Fourier transform can be defined. In Fig. 2(b),

$$
y(n)=\left\{\begin{array}{ll}
x\left(\frac{n}{L}\right) & \text { if } n=0, \pm L, \pm 2 L, \cdots \\
0 & \text { otherwise }
\end{array} \Longleftrightarrow Y(z)=X\left(z^{L}\right) .\right.
$$

The upsampler and downsampler are linear time-varying systems. Unless $x(n)$ is lowpass ${ }^{2}$ and bandlimited, downsampling results in aliasing and decimation thus requires an additional filter as in Fig. 3. This anti-aliasing filter $H(z)$ limits the bandwidth of $x(n)$. In Fig. 3,

$$
y(n)=\sum_{k=-\infty}^{+\infty} x(k) h(n M-k) .
$$

As upsampling causes imaging, interpolation requires a filter as in Fig. 4. This lowpass anti-imaging filter $H(z)$ removes the images and, as in Vaidyanathan (1993), we have

$$
y(n)=\sum_{k=-\infty}^{+\infty} x(k) h(n-k L)
$$

For $\operatorname{SRC}^{3}$ by a rational ratio $\frac{M}{L}$, interpolation by $L$ must be followed by decimation by $M$. Consequently, the cascade of the anti-imaging and anti-aliasing filters results in one filter, say $G(z)$, where the output, according to Vaidyanathan (1993), is

$$
y(n)=\sum_{k=-\infty}^{+\infty} x(k) g(n M-k L)
$$

Generally, $G(z)$ is a lowpass filter with a stopband edge at, as in Mitra (2006); Vaidyanathan (1993),

$$
\omega_{s} T=\min \left(\frac{\pi}{M}, \frac{\pi}{L}\right)=\frac{\pi}{\max (M, L)} .
$$

In practice, there is a roll-off factor $0 \leq \rho \leq 1$ so that $\omega_{s} T=\frac{\pi(1+\rho)}{\max (M, L)}$. If $M$ and $L$ are mutually coprime numbers, a decimator can be obtained by transposing the interpolator. The noble identities, defined as in Fig. 5, help move the filtering operations inside a multirate structure.

\footnotetext{
${ }^{2}$ This is not necessary to avoid aliasing. For example, if $X\left(e^{j \omega T}\right)$ is nonzero only at $\omega T \in\left[\omega_{1} T, \omega_{1} T+\frac{2 \pi}{M}\right]$ for some $\omega_{1} T$, there is no aliasing.

${ }^{3}$ If $L>M(L<M)$, we have interpolation (decimation) by a rational ratio $\frac{L}{M}>1\left(\frac{M}{L}>1\right)$. This chapter frequently refers to SRC by a rational ratio $R_{p}>1$.
} 


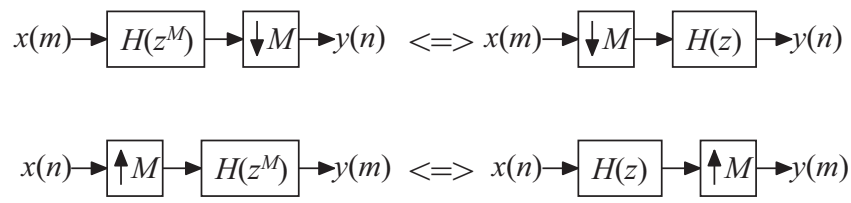

Fig. 5. Noble identities which allow us to move the arithmetic operations to the lower sampling frequency.

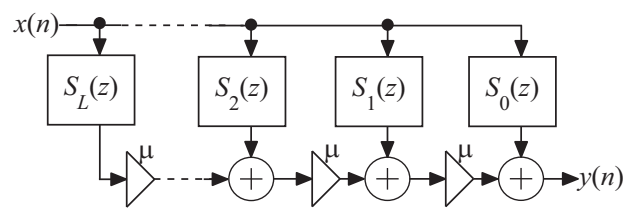

Fig. 6. Farrow structure with fixed subfilters $S_{k}(z)$ and variable fractional delay (FD) of $\mu$.

\subsection{Sampling rate conversion: Farrow structure}

In a conventional SRC and if the SRC ratio changes, we need new filters thereby reducing the flexibility. The Farrow structure, introduced in Farrow (1988) and shown in Fig. 6, obtains flexibility in an elegant way. The Farrow structure is traditionally composed of linear-phase finite-length impulse response (FIR) subfilters $S_{k}(z), k=0,1, \ldots, L$, with either a symmetric (for $k$ even) or antisymmetric (for $k$ odd) impulse response ${ }^{4}$.

When $S_{k}(z)$ are linear-phase FIR filters, the Farrow structure is sometimes referred to as the modified Farrow structure, e.g., Vesma \& Saramäki (1996), but we simply refer to it as the Farrow structure. The Farrow structure is efficient for interpolation whereas, for decimation, it is better to use the transposed Farrow structure, as discussed in Babic et al. (2002); Hentschel \& Fettweis (2000).

The transfer function of the Farrow structure is

$$
H(z, \mu)=\sum_{k=0}^{L} s_{k}(z) \mu^{k}=\sum_{k=0}^{L} \sum_{n=0}^{N_{k}} s_{k}(n) z^{-n} \mu^{k}=\sum_{n=0}^{N} \sum_{k=0}^{L} s_{k}(n) \mu^{k} z^{-n}=\sum_{n=0}^{N} h(n, \mu) z^{-n} .
$$

Here, $|\mu| \leq 0.5$ and $N$ is the order of the overall impulse response $h(n, \mu)$. Further, $\mu$ is the FD value, i.e., the time difference between each input sample and its corresponding output sample. In the rest of the chapter, we will use $h(n)$ and $H(z)$ instead of $h(n, \mu)$ and $H(z, \mu)$, respectively. If $\mu$ is constant for all input samples, the Farrow structure delays a bandlimited signal by a fixed $\mu$. In general, SRC amounts to delaying every input sample with a different $\mu$. Thus, by controlling $\mu$ for every input sample, the Farrow structure can perform SRC.

Generally, $S_{k}(z)$ are designed so that $H(z)$ approximates an allpass transfer function with FD of $\mu$ over the frequency range of interest according to Babic et al. (2002); Johansson \& Hermanowicz (2006); Johansson \& Löwenborg (2003); Pun et al. (2003); Tseng (2002); Vesma \& Saramäki (1996; 1997; 2000). The desired causal magnitude and unwrapped

\footnotetext{
${ }^{4}$ With infinite-length impulse response (IIR) filters, care must be taken to avoid transients as $\mu$ changes for every sample.
} 


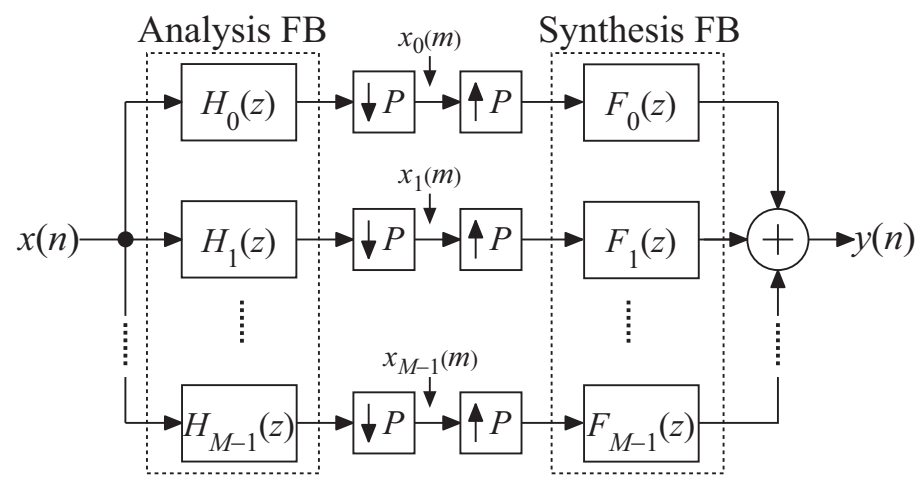

Fig. 7. General M-channel FB.

phase responses are

$$
\begin{aligned}
H_{\mathrm{des}}\left(e^{j \omega T}\right) & =e^{-j(\Delta+\mu) \omega T,} \\
\Phi_{\mathrm{des}}(\omega T) & =-(\Delta+\mu) \omega T,
\end{aligned}
$$

where

$$
\Delta=\frac{\max _{k}\left(N_{k}\right)}{2}
$$

The main advantage of the Farrow structure is its ability to perform rational SRC using only one set of $S_{k}(z)$ and by simple adjustments of $\mu$.

\subsection{General $M$-channel FBs}

An M-Channel filter bank (FB), shown in Fig. 7, splits $X(z)$ into the $M$ subbands $X_{m}(z), m=$ $0,1, \ldots, M-1$, using the analysis filter bank (AFB) filters $H_{m}(z)$. To reconstruct $X(z)$, we need the synthesis filter bank (SFB) filters $F_{m}(z)$. Furthermore, upsamplers and downsamplers by $P$ are also required as in Fig. 7. The output of a general $M$-channel FB is

$$
Y(z)=\frac{1}{P} \sum_{n=0}^{P-1} X\left(z W_{P}^{n}\right) \sum_{m=0}^{M-1} H_{m}\left(z W_{P}^{n}\right) F_{m}(z) .
$$

Ideally, $Y(z)$ is a scaled (by $\alpha$ ) and delayed (by $\beta$ ) version of $X(z)$, i.e., $y(n)=\alpha x(n-\beta)$. Such a system is referred to as PR. In near perfect reconstruction (NPR) FBs, $\alpha$ is frequency dependent and the distortion transfer function is

$$
V_{0}(z)=\frac{1}{P} \sum_{m=0}^{M-1} H_{m}(z) F_{m}(z),
$$

whereas the aliasing transfer functions are

$$
V_{l}(z)=\frac{1}{P} \sum_{m=0}^{M-1} H_{m}\left(z W_{P}^{l}\right) F_{m}(z), l=1,2, \ldots, P-1 .
$$


These FBs are generally linear periodic time-varying systems with a period $M$. Without aliasing, we have a linear time-invariant (LTI) system as defined in Vaidyanathan (1993). In a PR FB,

$$
\begin{aligned}
& V_{0}\left(e^{j \omega T}\right)=c, \quad c>0 \\
& V_{l}\left(e^{j \omega T}\right)=0, \quad l=1,2, \ldots, P-1 .
\end{aligned}
$$

If $P=M$, the FB is maximally decimated but $P<M$ leads to oversampled FBs as in Vaidyanathan (1993). If $V_{0}(z)$ is allpass (has linear-phase), we have zero amplitude (phase) distortion.

\subsection{Modulated FBs}

To obtain the AFB and SFB filters, one can modulate a single Nth-order linear-phase FIR prototype filter $G(z)=\sum_{n=0}^{N} g(n) z^{-n}$. With cosine modulation, which is outlined in Chen \& Chiueh (2008); Ihalainen et al. (2007); Saramäki \& Bregović (2001a), we have

$$
\begin{aligned}
& h_{m}(n)=2 g(n) \cos \left[(m+0.5) \frac{\pi}{M}\left(N-n+\frac{M+1}{2}\right)\right], \\
& f_{m}(n)=2 g(n) \cos \left[(m+0.5) \frac{\pi}{M}\left(n+\frac{M+1}{2}\right)\right]=h_{m}(N-n) .
\end{aligned}
$$

In a PR cosine modulated filter bank (CMFB), $N=2 K M-1$ where $K$ is an integer overlapping factor as defined in Viholainen et al. (2006). For complex modulated FBs,

$$
\begin{aligned}
& h_{m}(n)=g(n) W_{M}^{-m n}, \\
& f_{m}(n)=h_{m}(n) .
\end{aligned}
$$

In the maximally decimated case, we can use modified discrete Fourier transform (MDFT) FBs, as in Bregović \& Saramäki (2005); Karp \& Fliege (1999). An M-channel MDFT FB can be realized as either of the following, according to Fliege (1995),

- Two SRC stages with ratios $\frac{M}{2}$ and 2 while adding some phase offset between these stages.

- Two separate FBs where the phase offset is applied outside the AFB and SFB.

If an MDFT FB is PR, $N$ is an integer as $K M+s$ where $0 \leq s<M$. With the AFB and SFB filters, having uniform or nonuniform passbands, we have uniform or nonuniform FBs as in Vaidyanathan (1993). Nonuniform FBs can also be obtained by modulation, as discussed in Princen (1994), where

$$
\begin{aligned}
& h_{m}(n)=a_{m} g_{m}(n) e^{-\frac{j \pi \alpha_{m}}{M_{m}}\left(n-\frac{L_{m}-1}{2}\right)}+a_{m}^{*} g_{m}^{*}(n) e^{\frac{j \pi \alpha_{m}}{M_{m}}\left(n-\frac{L_{m}-1}{2}\right)}, \\
& f_{m}(n)=b_{m} g_{m}(n) e^{-\frac{j \pi \alpha_{m}}{M_{m}}\left(n-\frac{L_{m}-1}{2}\right)}+a_{m}^{*} g_{m}^{*}(n) e^{\frac{j \pi \alpha_{m}}{M_{m}}\left(n-\frac{L_{m}-1}{2}\right)} .
\end{aligned}
$$

Here, $\alpha_{m}=\left(K_{m}+0.5\right)$ and $g_{m}(n)$ is the (possibly complex) prototype filter of length $L_{m}$ with $M_{m}$ being the decimation factor in each branch. Each branch has a center frequency as $\pm \frac{\pi \alpha_{m}}{M_{m}}$ with $K_{m}$ being an integer where $a_{m}$ and $b_{m}$ define the modulation phase. The nonuniform FBs achieve a more general time and frequency tiling. The sine modulated filter bank (SMFB) is obtained similar to (16) and (17). The exponentially modulated filter bank (EMFB), with 


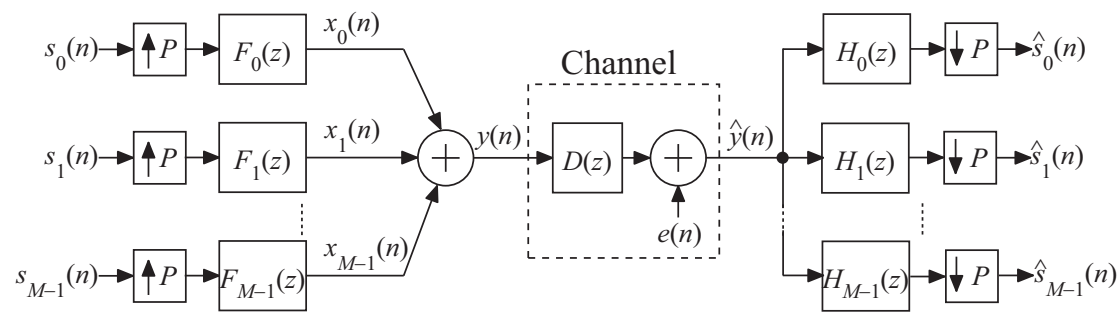

Fig. 8. General M-channel TMUX.

complex filters, is a combination of SMFB and CMFB, as outlined in Chen \& Chiueh (2008); Ihalainen et al. (2007).

\subsubsection{Filter design for modulated FBs}

To design the prototype filter $G(z)$, we can use any standard filter design technique, e.g., Bregović \& Saramäki (2005); Fliege (1995); Heller et al. (1999); Martin-Martin et al. (2008; 2005); Mirabbasi \& Martin (2003); Mitra (2006); Saramäki \& Bregović (2001a); Saramäki \& Bregovic (2001b); Vaidyanathan (1993); Viholainen et al. (1999). The MDFT FB has a lowpass $G(z)$ with a stopband edge at $\omega_{s} T=\frac{2 \pi}{M}$ according to Fliege (1995). The CMFB has a lowpass $G(z)$ with a stopband edge at $\omega_{s} T=\frac{\pi(1+\rho)}{2 M}$ and a 3-dB cutoff frequency at $\omega T=\frac{\pi}{2 M}$ as discussed in Diniz et al. (2004); Martin-Martin et al. (2005). If $0<\rho \leq 1$, only the adjacent branches overlap. With $1<\rho \leq 2$ (or $\rho>2$ ), two (or at least three) adjacent branches overlap. In both FBs, $G(z)$ satisfies the power complementary property.

\subsection{General M-channel TMUXs}

A transmultiplexer (TMUX) converts the time multiplexed components of a signal into a frequency multiplexed version and back so that several users transmit and receive over a common channel, as noted in Vaidyanathan \& Vrcelj (2004). A TMUX is also referred to as a FB transceiver, e.g., Beaulieu \& Champagne (2009); Bianchi \& Argenti (2007); Borna \& Davidson (2007); Chiang et al. (2007); Lin \& Phoong (2001).

Assume that we want to transmit a series of symbol streams $s_{k}(n), k=0,1, \ldots, M-1$, through a channel. As in Fig. 8, we can pass $s_{k}(n)$ through the transmitter filters $F_{k}(z)$. Then, (4) gives

$$
x_{k}(n)=\sum_{m=-\infty}^{\infty} s_{k}(m) f_{k}(n-m P) .
$$

Here, the channel is described by a possibly complex LTI filter $D(z)=\sum_{n=0}^{L_{D}} d(n) z^{-n}$ followed by an additive noise source $e(n)$. The receiver filters $H_{k}(z)$ separate the signals and only a downsampling by $P$ is needed to retrieve the original symbol streams. Ignoring the channel and for $i=0,1, \ldots, M-1$, we have

$$
\hat{S}_{i}(z)=\sum_{k=0}^{M-1} S_{k}(z) T_{k i}\left(z^{P}\right), \quad T_{k i}\left(z^{P}\right)=\frac{1}{P} \sum_{l=0}^{P-1} F_{k}\left(z W_{P}^{l}\right) H_{i}\left(z W_{P}^{l}\right) .
$$




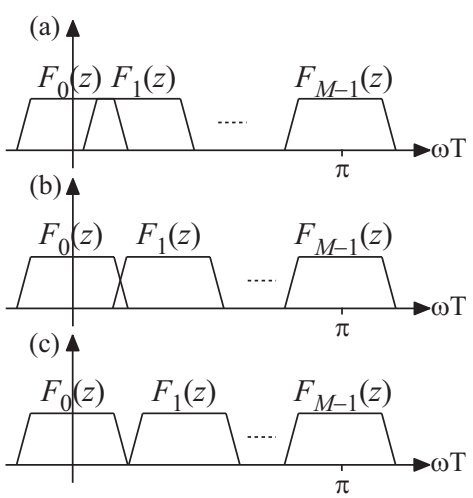

Fig. 9. M-channel TMUX filters. (a) Overlapping. (b) Marginally overlapping. (c) Non-overlapping.

Typical characteristics of $F_{k}(z)$ and $H_{k}(z)$ are shown in Fig. 9. Similar to FBs, TMUXs can be redundant $(P>M)$ or critically sampled $(P=M)$. To avoid inter-symbol interference (ISI), a level of redundancy may be needed such that $P-M \geq L_{D}$, according to Bianchi et al. (2005). The output of the TMUX in (23) is

$$
\hat{S}_{i}(z)=T_{i i}(z) S_{i}(z)+\sum_{k=0, k \neq i}^{P-1} T_{k i}(z) S_{k}(z)
$$

where $T_{i i}(z)$ and $T_{k i}(z)$ represent the ISI and the inter-carrier interference (ICI), respectively, as in Furtado et al. (2005). The ISI (ICI) is sometimes also referred to as interband (cross-band) ISI, e.g., Chiang et al. (2007).

If an LTI filter is placed between an upsampler and a downsampler of ratio $P$, the overall system is equivalent to the decimated (by $P$ ) version of its impulse response, as mentioned in Vaidyanathan (1993). In this case, designing $F_{k}(z)$ and $H_{k}(z)$ so that the decimated (by $P$ ) version of $F_{k}(z) H_{m}(z)$ becomes a pure delay if $k=m$ and zero otherwise, the TMUX becomes PR. In terms of (24), this means

$$
\begin{aligned}
& T_{i i}(z)=\frac{1}{P} \sum_{l=0}^{P-1} F_{i}\left(z^{\frac{1}{P}} W_{P}^{l}\right) H_{i}\left(z^{\frac{1}{P}} W_{P}^{l}\right)=\alpha z^{-\beta}, \\
& T_{k i}(z)=\frac{1}{P} \sum_{l=0}^{P-1} F_{k}\left(z^{\frac{1}{P}} W_{P}^{l}\right) H_{i}\left(z^{\frac{1}{P}} W_{P}^{l}\right)=0 .
\end{aligned}
$$

In a PR system, $\hat{s}_{k}(n)=\alpha s_{k}(n-\beta)$. The PR properties can be satisfied for both critically sampled and redundant TMUXs. For the critically sampled case, there may not always exist FIR or stable IIR solutions. Therefore, some redundancy makes the solutions feasible, as in de Barcellos et al. (2006); Kovačević \& Vetterli (1993); Li et al. (1997); Xie, Chan \& Yuk (2005); Xie, Chen \& Sho (2005), and it also simplifies the PR conditions. Duality of TMUXs and FBs allows one to obtain a TMUX from its corresponding FB, as noted in Vaidyanathan (1993). 


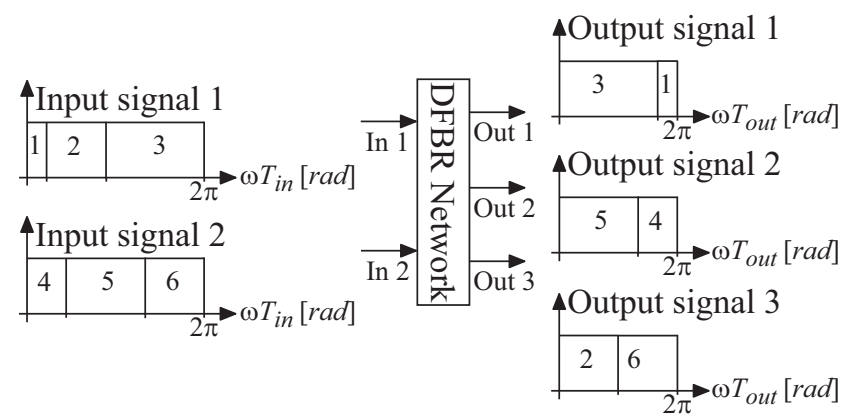

Fig. 10. Approach I: DFBR networks process composite signals to reallocate users from one composite input signal to another composite output signal.

This duality applies to both critically sampled and redundant systems. If a FB is free from aliasing, the corresponding TMUX is free from ICI, according to Fliege (1995).

\section{Approach I: use of DFBR networks}

For DFBR, we assume that signals from several users, e.g., mobile handsets in a cellular network or computers in a wireless local area network (WLAN), have been added into a composite signal at a main station, e.g., a base station in a cellular network or an access point in a WLAN. This main station then finds available frequency slots and reallocates each user to one of them. Such a main station is similar to a bentpipe satellite payload, as outlined in Nguyen et al. (2002), with its idea of operation shown in Fig. 10. The composite signals are processed by the DFBR network and the users are reallocated to new frequency slots. These slots could be different antenna beams of a satellite payload or different cells in a cellular network. Multiple antennas of a satellite payload perform signal filtering in spatial rather than frequency domain. This is similar to the techniques utilizing multiple antennas for cognitive radios which are discussed in Cabric \& Brodersen (2005). The DFBR networks could also be useful for the centralized cooperative cognitive radios, as in Ganesan \& Li (2005), and they can also be considered as secondary base stations in licensed band cognitive radios. In licensed band networks, the DFBR can coexist with the primary networks so as to opportunistically operate in an overlay transmission.

The DFBR network can be a mutli-input multi-output system as it can have a number of composite input and output signals. The dynamic nature of the DFBR networks allows the users to occupy any suitable ${ }^{5}$ frequency slot in a time-varying manner. Each user can be sent in contiguous or separate frequency bands leading to contiguous or fragmented DFBR which is outlined in Leaves et al. (2004). The separate frequency bands can be considered as a multi-spectrum transmission. Specifically, as white spaces are mostly fragmented, according to Yuan, Bahl, Chandra, Chou, Ferrell, Moscibroda, Narlanka \& Wu (2007), the user signals can be transmitted in several non-contiguous frequency bands.

\footnotetext{
${ }^{5}$ The frequency slot depends on spatial and temporal parameters, e.g., the number of available slots, user movement, and primary user activity, etc. Akyildiz et al. (2006) but the DFBR network is independent of these parameters.
} 

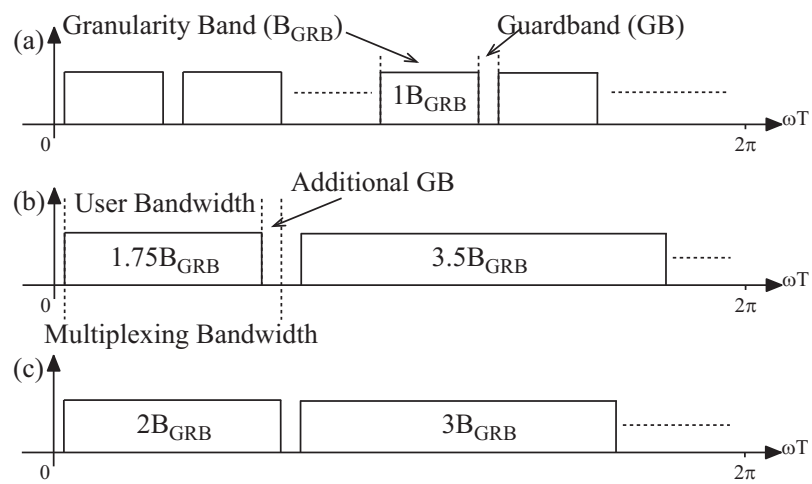

Fig. 11. User bandwidth versus multiplexing bandwidth. The multiplexing bandwidth is an integer multiple of the GRB and it contains a user bandwidth as well as some additional GB.

\subsection{Structure of the DFBR network}

This chapter uses the term DFBR which is essentially the same as the flexible frequency-band reallocation (FFBR) in, e.g., Abdulazim \& Göckler (2006); Eghbali et al. (2009a); Johansson \& Löwenborg (2007); Rosenbaum et al. (2006). For the illustrations, we will use the FFBR network, in Johansson \& Löwenborg (2007), but one can use any other FFBR network as well.

\subsection{User bandwidth versus multiplexing bandwidth}

The DFBR networks divide the user signals into a number of granularity bands (GRBs) on which the frequency shifts are performed. As the DFBR networks utilize FBs, the multiplexing bandwidth must be an integer multiple of the granularity band (GRB). The DFBR networks perform frequency shifts on users whose bandwidths are, in general, rational multiples of the GRB. An important issue is to ensure that the users do not share a GRB. This can be achieved by allowing some additional guardband (GB). However, the additional GB affects the spectrum efficiency resulting in a trade-off. As in Fig. 11, a multiplexing bandwidth contains a user bandwidth and some additional GB.

\subsection{Reconfigurability}

A cognitive radio should adjust its operating parameters without hardware modifications as discussed in Jondral (2005). It is built on the platform for a software defined radio where the processing is mainly in the digital domain, according to Zhao \& Sadler (2007). There are several reconfigurable parameters such as operating frequency, modulation method, transmission power, and communication standard etc. For adaptable operating frequency, or flexible frequency carrier tuning, as in Leaves et al. (2004), a cognitive radio should change its operating frequency without restricting the system throughput and hardware.

The DFBR networks can perform any frequency shift of any user having any bandwidth, using a channel switch. This switch seamlessly directs different FB channels to their desired outputs without any arithmetic complexity. In addition, the system parameters are determined and fixed only once, offline. The reconfigurable operation is then performed by reconfiguring 
(a)

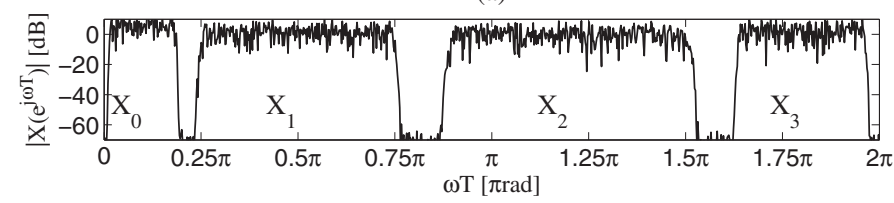

(b)

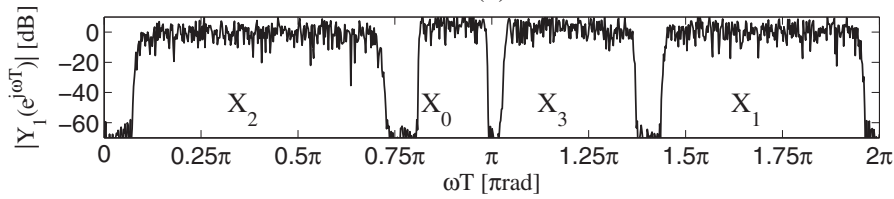

(c)

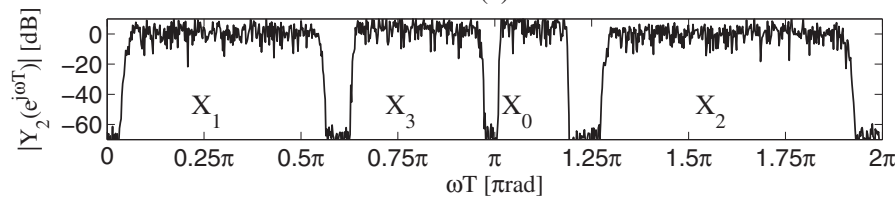

Fig. 12. Input and the reallocated outputs using the channel switch configurations in Figs. 14(a) and 14(b).

(a)

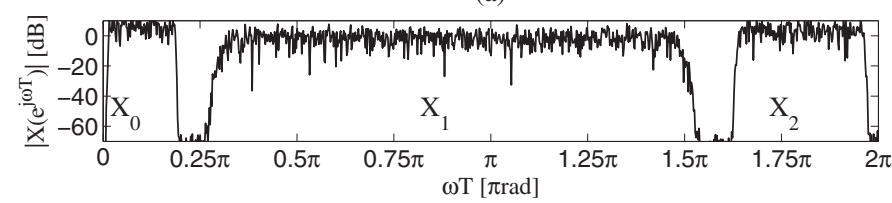

(b)

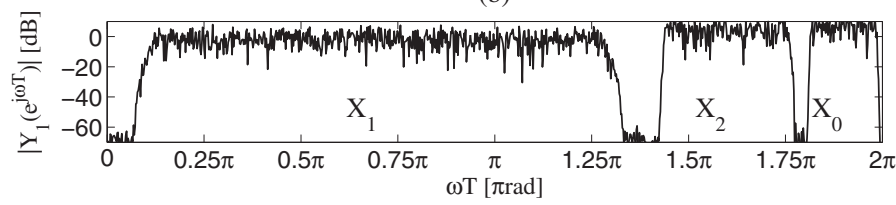

(c)

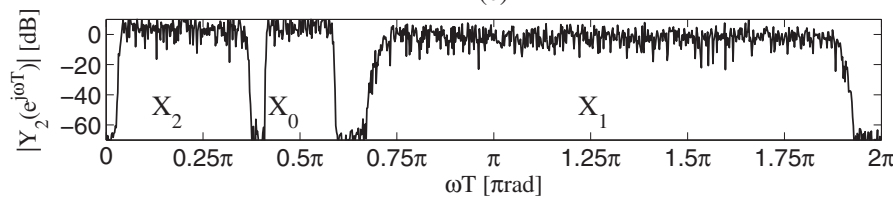

Fig. 13. Input and the reallocated outputs using the channel switch configurations in Figs. 14(c) and 14(d). 


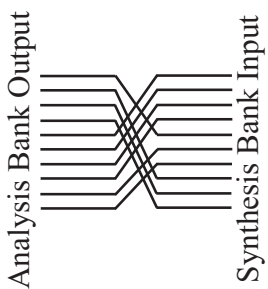

(a) Scenario I.

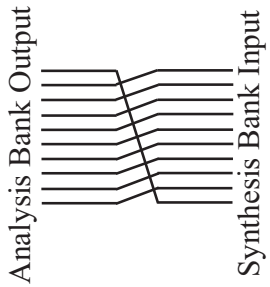

(c) Scenario III.

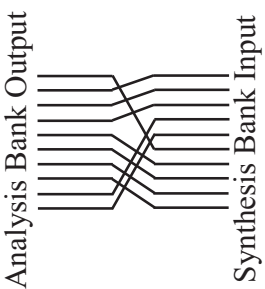

(b) Scenario II.

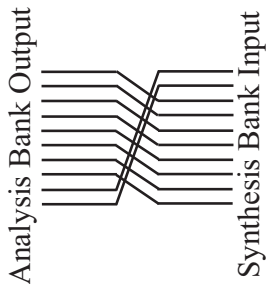

(d) Scenario IV.

Fig. 14

the channel switch, online. Here, the user bandwidths are predetermined but they can be arbitrary. The DFBR network makes a hand off by changing the operating frequency.

Figures 12 and 13 show two cases where, respectively, four and three users have occupied the whole frequency band. To generate these user signals, the multimode TMUX of Eghbali et al. (2008b) has been used. In Fig. 12(a), the user signals $\left\{X_{0}, X_{1}, X_{2}, X_{3}\right\}$ occupy, respectively, user bandwidths of $\{1,2.9,3.6,1.9\}$ GRBs. Each GRB has a spectral width of $\frac{2 \pi}{Q}-\frac{2 \epsilon \pi}{Q}$ with $Q=10$ and $\epsilon=0.125$. In Fig. 13(a), the user signals $\left\{X_{0}, X_{1}, X_{2}\right\}$ occupy $\{1,6.9,1.9\}$ GRBs, respectively. To ensure that the users do not share a GRB, one can add some additional GB. This difference in the amount of the GB, between different users, can be recognized from Figs. 12 and 13.

These examples assume the DFBR network to operate on the same antenna beam. By having several DFBR networks, the users can be reallocated between different antenna beams according to Johansson \& Löwenborg (2007). This requires a duplication of DFBR networks and a channel switch which directs the user signals between different DFBR networks. Each branch of the channel switches, in Figs. 14(a)-14(d), represents the operation of two FB channels as each GRB contains two FB channels. Specifically, the values of $N, M$, and $L$, in Johansson \& Löwenborg (2007), are 20, 10, and 2, respectively, for the examples above.

\subsection{Modifications}

The use of DFBR networks in cognitive radios needs some modifications, mainly in the system parameters. For different system parameters, the implementation complexity may be different but once the parameters are chosen, the implementation complexity remains constant and the system can be easily reconfigured on the same hardware platform. For the DFBR networks, the width of a GRB must be proportional to that of the spectrum holes. Thus, one requires to 


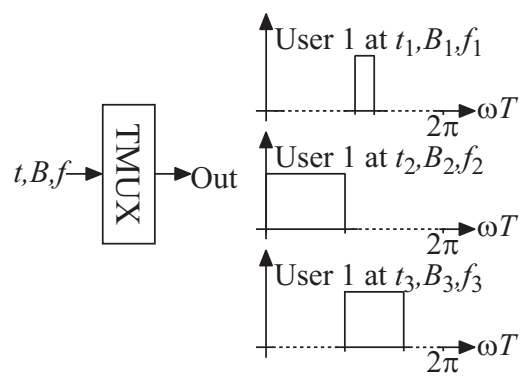

Fig. 15. Approach II: TMUXs to perform DFBA. At any time $t_{k}$, each user decides its bandwidth $B_{k}$ and operating frequency $f_{k}$.

choose a value for the $B_{G R B}=\frac{2 \pi(1-\epsilon)}{Q}$, in Fig. 11, so as to represent any spectrum hole as a rational multiple of $B_{G R B}$.

\section{Approach II: use of TMUXs}

Using TMUXs, each user terminal can adjust its operating frequency and bandwidth. The basic idea is depicted in Fig. 15 where different bandwidths and center frequencies can be generated using multirate signal processing techniques. These TMUXs can also be regarded as the time-spectrum blocks, discussed in Yuan, Bahl, Chandra, Moscibroda, Narlanka \& Wu (2007), which can transmit any amount of data at any time interval and on any portion of the frequency spectrum. This applies if the licensed users choose frequency division multiple access and/or time division multiple access as their spectrum access mode. Then, the spectrum holes are identified in the time/frequency plane, as outlined in Jondral (2007). As shown in Fig. 16, the interpolation part represents the transmitter with a variable filter placing the desired user signal at the desired center frequency. The receiver, i.e., the decimation part, retrieves the input signal.

Similar to straightforward DFBR solutions, one can use conventional nonuniform TMUXs to place users, with different bandwidths, at different center frequencies. This becomes inefficient when simultaneously considering the increased number of communication scenarios and the desire to support dynamic communications. In this context, TMUX structures of the general form shown in Fig. 17 can be used. In the SFB, the system $C_{p}$ performs interpolation by a rational ratio $R_{p}$ whereas the system $\hat{C}_{p}$ in the AFB performs decimation by a rational ratio $R_{p}$.

\subsection{Structure of the TMUX}

Any of the TMUXs, in the references Eghbali et al. (2007a; 2008a;b; 2009b; 2010); Eghbali, Johansson \& Löwenborg (2011a;b), can be used here. The TMUX, in Eghbali et al. (2010); Eghbali, Johansson \& Löwenborg (2011b), has a rather different structure. Instead of variable lowpass filters and frequency shifters as, in Eghbali et al. (2007a; 2008a;b; 2009b); Eghbali, Johansson \& Löwenborg (2011a), it performs bandpass rational SRC using flexible commutators and fixed bandpass filters. However, one can generally describe it in terms of Fig. 17. 


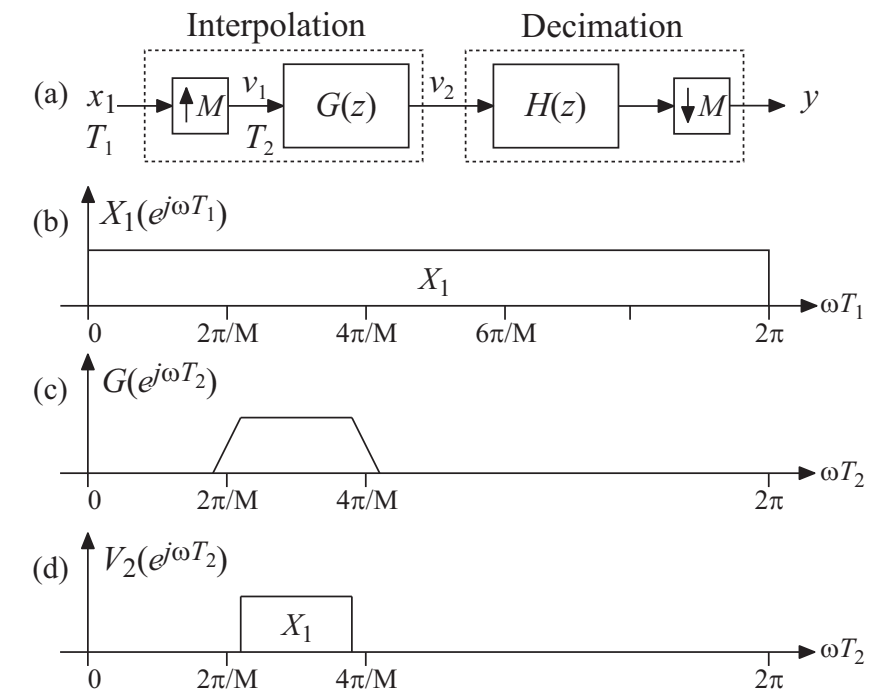

Fig. 16. Principle of TMUXs using multirate building blocks. The interpolation (decimation) part represents the transmitter (receiver). Variable filters place the desired user signal at the desired center frequency.

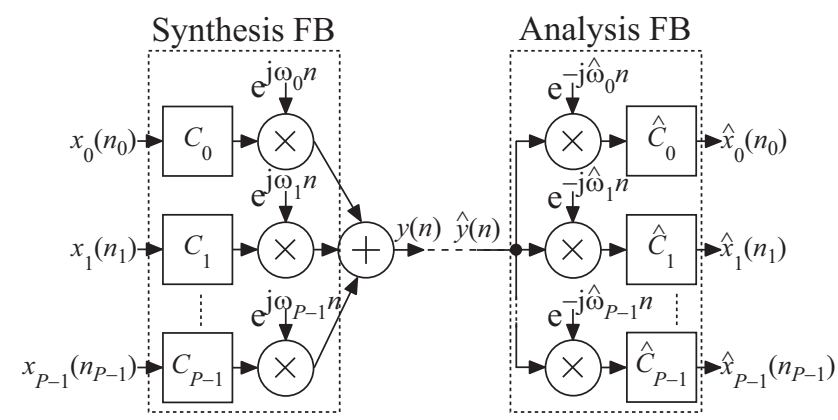

Fig. 17. General structure of a multimode TMUX where systems $C_{p}$ and $\hat{C}_{p}$ perform rational SRC.

\subsection{Reconfigurability}

A cognitive radio must adjust its operating frequency and bandwidth without hardware modifications. The DFBR networks partially provide this capability but they have no control over the user bandwidth. In contrast, the TMUX-based approaches add reconfigurability to the user bandwidth as well. Furthermore, they bring flexible receiver signal filtering, outlined in Leaves et al. (2004), by changing the transmitter and receiver filters. As can be seen from Figs. 12 and 13, the TMUX allows different numbers of users, e.g., four and three, with different user bandwidths to occupy the whole frequency band. These TMUXs provide this full reconfigurability without any hardware changes. 


\subsection{Modifications}

Similar to the DFBR networks, we should have certain system parameters to eliminate the need for hardware changes while having simple reconfigurability. Regarding DFBA, there are different ways to perform SRC which could be useful in different scenarios. The TMUX, in Eghbali et al. (2007a; 2008b), generates a GRB through integer interpolation by, e.g., $W$, thereby resulting in $B_{G R B}=\frac{2 \pi(1+\rho)}{W}$ where $\rho$ is the roll-off. Then, rational $R_{p}$ multiples of $B_{G R B}$ can be created using the Farrow structure.

The TMUX, in Eghbali et al. (2008a; 2009b); Eghbali, Johansson \& Löwenborg (2011a), assumes no GRBs and it allows the users to occupy any portion of the spectrum. It utilizes the Farrow structure to perform general rational SRC by, e.g., $R_{p}=\frac{A_{p}}{B_{p}}$. Here, one can also assume a GRB of size $B_{G R B}=\frac{2 \pi(1+\rho)}{A_{p}}$. Then, users can have bandwidths which are integer $B_{p}$ multiples of $B_{G R B}$.

Although references Eghbali et al. (2010); Eghbali, Johansson \& Löwenborg (2011b) propose a slightly different TMUX, one can also assume $B_{G R B}=\frac{2 \pi(1+\rho)}{M}$. Then, users have bandwidths which are integer $M_{p}$ multiples of $B_{G R B}$. This applies to the case with MDFT FBs but for a $\mathrm{CMFB}$, similar formulae can be derived.

\section{Choice of frequency shifters}

To perform a hand off without information loss, the DFBR network requires the users not to share a GRB. Consequently, a lossless reallocation requires to (i) generate appropriate frequency division multiplexed (FDM) input patterns, and (ii) determine proper parameters for the DFBR networks. To generate the input patterns, the reconfigurability of the TMUXs in Fig. 17 can be used. After generating the user signals with desired bandwidths, the frequency shifters $\omega_{p}, p=0,1 \ldots, P-1$, can be computed to allow some additional GB. Here, an example using the TMUX in Eghbali et al. (2008b) is provided. Assuming some bandwidths which are rational, e.g., $R_{p}$, multiples of $B_{G R B}$, the subcarrier $\omega_{p}$ for user $p$ is

$$
\omega_{p}= \begin{cases}\frac{F_{0}}{2} & \text { if } p=0 \\ \sum_{k=0}^{p-1} F_{k}+\frac{F_{p}}{2} & \text { if } p \neq 0 .\end{cases}
$$

where $F_{p}=\left\lceil R_{p}\right\rceil \frac{2 \pi}{Q}, p=0,1, \ldots, k$, with $\lceil$.$\rceil being the ceiling operation. Here, F_{p}$ is the multiplexing bandwidth and the ceiling operation ensures that the users do not share a GRB. This formulation applies to the case where DFBA and DFBR are simultaneously used. Otherwise, similar formulae can be used but one may anyhow require some additional GB due to the design margins.

In Figs. 12 and 13, the users occupy $R_{p}=\{2.9,3.6,1.9,6.9\}$ GRBs. This necessitates an additional GB which is $E_{p}=\{0.1,0.4,0.1,0.1\}$ multiples of $\frac{2 \pi}{Q}$. Therefore, the spectrum efficiency decreases. For a set of values $R_{p}, p=0,1, \ldots, P-1$, about

$$
\eta_{\text {dec }}=\frac{\frac{2 \pi}{Q} \sum_{p=0}^{P-1}\left(\left\lceil R_{p}\right\rceil-R_{p}\right)}{2 \pi}=\frac{\sum_{p=0}^{P-1}\left\lceil R_{p}\right\rceil-R_{p}}{Q}
$$


(a)

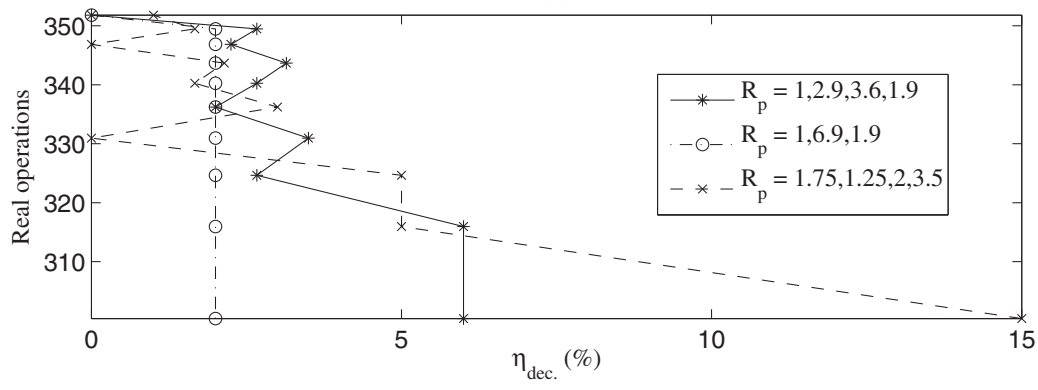

(b)

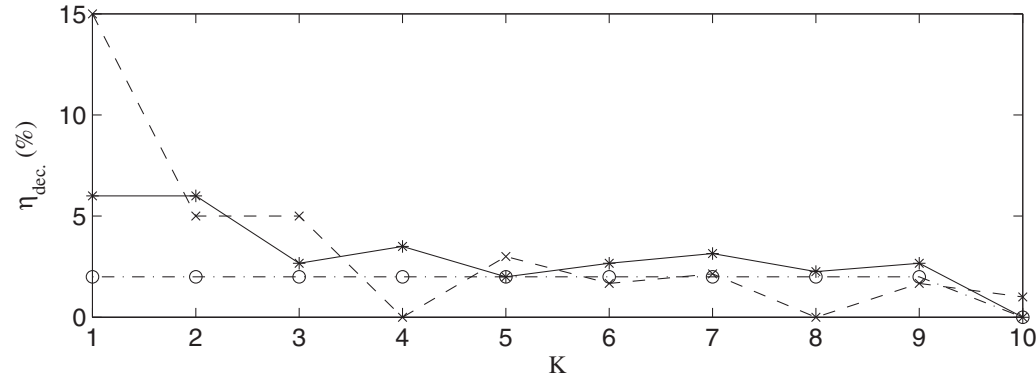

Fig. 18. Trade-off between spectrum efficiency and arithmetic complexity. (a) Decrease in spectrum efficiency versus per-sample arithmetic complexity. (b) Trend of spectrum efficiency versus different $K$ in (29).

percent of the spectrum in $[0,2 \pi]$ is not used. In the examples of Figs. 12 and 13, about $6 \%$ and $2 \%$ of the total spectrum is not used due to the additional GB. To decrease $\eta_{d e c}$, one can increase $Q$ by, e.g., $K$ times, so that (28) becomes

$$
\eta_{\text {dec }}=\frac{\sum_{p=0}^{P-1}\left\lceil K R_{p}\right\rceil-K R_{p}}{K Q} .
$$

However, increasing $Q$ would increase the order of the prototype filter of the corresponding FB. For each $K$, the prototype filter of the DFBR network would have a transition band of $\frac{2 \pi \epsilon}{K Q}$ according to Eghbali et al. (2009a); Johansson \& Löwenborg (2007). As the order of a linear-phase FIR filter is inversely proportional to the width of its transition band, there is a trade-off between the spectrum efficiency and the arithmetic complexity.

With a $K$-fold increase in $Q$, the length of the prototype filter and the number of FB channels increase proportional to $K$. Figure 18 shows the trend in spectrum efficiency with respect to the per-sample arithmetic complexity of the DFBR network in Johansson \& Löwenborg (2007). Here, the examples of Figs. 12 and 13 as well as that of Eghbali et al. (2008b) with $R_{p}=\{1.75,1.25,2,3.5\}$ are considered. As can be seen, a larger $K$ increases the per-sample arithmetic complexity but it decreases $\eta_{d e c}$. The values of $R_{p}$ mainly determine the maximum and minimum amounts of $\eta_{d e c}$. Hence, for every set of $R_{p}$, one can determine a $K$ such that $\eta_{\text {dec }}$ and the per-sample arithmetic complexity are within the acceptable ranges. 


\section{Conclusion}

This chapter discussed two approaches for the baseband processing in cognitive radios based on DFBR and DFBA. They can support different bandwidths and center frequencies for a large set of users while being easily reconfigurable.

In DFBR networks, composite FDM signals are processed and the users are reallocated to new center frequencies. They are applicable to cognitive radios with multiple antennas, centralized cooperative cognitive radios, and secondary base stations in licensed band cognitive radios. In DFBA networks, each user controls its operating frequency and bandwidth. These networks can be regarded as the time-spectrum blocks.

The reconfigurability of DFBA and DFBR is performed either by a channel switch, in DFBR, or by variable multipliers/commutators, in DFBA. The examples in Figs. 12 and 13 show the increased flexibility to allocate and reallocate any user to any center frequency by simultaneous utilization of DFBA and DFBR. In this case, the individual users can occupy any available frequency slot and be reallocated by the base station.

Basically, utilizing any of Approaches I and II only requires modifications imposed by the special choice of the system parameters. After choosing these parameters once, we must design the filters to satisfy any desired level of error. Then, the same hardware can be reconfigured in a simple manner.

\section{References}

Abdulazim, M. N. \& Göckler, H. G. (2005a). Design options of the versatile two-channel SBC-FDFMUX filter bank, Proc. Eur. Conf. Circuit Theory Design, Cork, Ireland.

Abdulazim, M. N. \& Göckler, H. G. (2005b). Efficient digital on-board de- and remultiplexing of FDM signals allowing for flexible bandwidth allocation, Proc. Int. Commun. Satellite Syst. Conf., Rome, Italy.

Abdulazim, M. N. \& Göckler, H. G. (2006). Flexible bandwidth reallocation MDFT SBC-FDFMUX filter bank for future bent-pipe FDM satellite systems, Proc. Int. Workshop Signal Processing Space Commun., Noordwijk, Netherlands.

Abdulazim, M. N., Kurbiel, T. \& Göckler, H. G. (2007). Modified DFT SBC-FDFMUX filter bank systems for flexible frequency reallocation, Proc. Eur. Signal Processing Conf., Poznan, Poland, pp. 60-64.

Akyildiz, I. F., Lee, W. Y., Vuran, M. C. \& Mohanty, S. (2006). Next generation/dynamic spectrum access/cognitive radio wireless networks: A survey, Comput. Networks J. (Elsevier) 50: 2127-2159.

Akyildiz, I. F., Lee, W. Y., Vuran, M. C. \& Mohanty, S. (2008). A survey on spectrum management in cognitive radio networks, IEEE Commun. Mag. 46(4): 40-48.

Arbesser-Rastburg, B., Bellini, R., Coromina, F., Gaudenzi, R. D., del Rio, O., Hollreiser, M., Rinaldo, R., Rinous, P. \& Roederer, A. (2002). R\&D directions for next generation broadband multimedia systems: An ESA perspective, Proc. AIAA Int. Commun. Satellite Syst. Conf. Exhibit, Montreal, Canada.

Babic, D., Vesma, J., Saramäki, T. \& Renfors, M. (2002). Implementation of the transposed Farrow structure, Proc. IEEE Int. Symp. Circuits Syst., Vol. 4, pp. 5-8. 
Beaulieu, F. D. \& Champagne, B. (2009). Design of prototype filters for perfect reconstruction DFT filter bank transceivers, Signal Processing 89(1): 87-98.

Bianchi, T. \& Argenti, F. (2007). SVD-Based techniques for zero-padded block transmission over fading channels, IEEE Trans. Signal Processing 55(2): 594-604.

Bianchi, T., Argenti, F. \& Re, E. D. (2005). Performance of filterbank and wavelet transceivers in the presence of carrier frequency offset, IEEE Trans. Commun. 53(8): 1323-1332.

Borna, B. \& Davidson, T. N. (2007). Biwindowed discrete multitone transceiver design, IEEE Trans. Signal Processing 55(8): 4217-4226.

Bregović, R. \& Saramäki, T. (2005). A systematic technique for designing linear-phase FIR prototype filters for perfect-reconstruction cosine-modulated and modified DFT filter banks, IEEE Trans. Signal Processing 53(8): 3193-3201.

Cabric, D. \& Brodersen, R. W. (2005). Physical layer design issues unique to cognitive radio systems, Proc. IEEE Personal Indoor Mobile Radio Commun.

Cabric, D., ÓDonnell, I. D., Chen, M. S. W. \& Brodersen, R. W. (2006). Spectrum sharing radios, IEEE Circuits Syst. Mag. 6(2): 30-45.

Chen, K.-H. \& Chiueh, T.-D. (2008). A cognitive radio system using discrete wavelet multitone modulation, IEEE Trans. Circuits Syst. I 55(10): 3246-3258.

Chiang, H.-T., Phoong, S.-M. \& Lin, Y.-P. (2007). Design of nonuniform filter bank transceivers for frequency selective channels, EURASIP J. Adv. Signal Processing 2007, Article ID 61396.

de Barcellos, L. C. R., Diniz, P. S. R. \& Netto, S. L. (2006). A generalized oversampled structure for cosine-modulated transmultiplexers and filter banks, Circuits Syst. Signal Processing 25(2): 131-151.

Devroye, N., Mitran, P. \& Tarokh, V. (2006). Achievable rates in cognitive radio channels, IEEE Trans. Inform. Theory 52(5): 1813-1827.

Diniz, P. S. R., de Barcellos, L. C. R. \& Netto, S. L. (2004). Design of high-resolution cosine-modulated transmultiplexers with sharp transition band, IEEE Trans. Signal Processing 52(5): 1278-1288.

Eghbali, A., Johansson, H. \& Löwenborg, P. (2007a). An arbitrary bandwidth transmultiplexer and its application to flexible frequency-band reallocation networks, Proc. Eur. Conf. Circuit Theory Design, Seville, Spain, pp. 248-251.

Eghbali, A., Johansson, H. \& Löwenborg, P. (2007b). Flexible frequency-band reallocation MIMO networks for real signals, Proc. Int. Symp. Image Signal Processing Anal., Istanbul, Turkey, pp. 75-80.

Eghbali, A., Johansson, H. \& Löwenborg, P. (2008a). A Farrow-structure-based multi-mode transmultiplexer, Proc. IEEE Int. Symp. Circuits Syst., Seattle, Washington, USA, pp. 3114-3117.

Eghbali, A., Johansson, H. \& Löwenborg, P. (2008b). A multimode transmultiplexer structure, IEEE Trans. Circuits Syst. II 55(3): 279-283.

Eghbali, A., Johansson, H. \& Löwenborg, P. (2009a). Flexible frequency-band reallocation: complex versus real, Circuits Syst. Signal Processing 28(3): 409-431.

Eghbali, A., Johansson, H. \& Löwenborg, P. (2009b). On the filter design for a class of multimode transmultiplexers, Proc. IEEE Int. Symp. Circuits Syst., Taipei, Taiwan, pp. 89-92. 
Eghbali, A., Johansson, H. \& Löwenborg, P. (2010). Reconfigurable nonuniform transmultiplexers based on uniform filter banks, Proc. IEEE Int. Symp. Circuits Syst., Paris, France, pp. 2123-2126.

Eghbali, A., Johansson, H. \& Löwenborg, P. (2011a). A class of multimode transmultiplexers based on the Farrow structure, Circuits Syst. Signal Processing . accepted.

Eghbali, A., Johansson, H. \& Löwenborg, P. (2011b). Reconfigurable nonuniform transmultiplexers using uniform modulated filter banks, IEEE Trans. Circuits Syst. I 58(3): 539-547.

Eghbali, A., Johansson, H., Löwenborg, P. \& Göckler, H. G. (2011). Flexible dynamic frequency-band reallocation and allocation: from satellite-based communication systems to cognitive radios, J. Signal Processing Syst. 62(2): 187-203.

Evans, B., Werner, M., Lutz, E., Bousquet, M., Corazza, G. E., Maral, G. \& Rumeau, R. (2005). Integration of satellite and terrestrial systems in future multimedia communications, IEEE Wireless Commun. Mag. 12(5): 72-80.

Farrow, C. W. (1988). A continuously variable digital delay element, Proc. IEEE Int. Symp. Circuits Syst., Vol. 3, Espoo, Finland, pp. 2641-2645.

Farserotu, J. \& Prasad, R. (2000). A survey of future broadband multimedia satellite systems, issues and trends, IEEE Commun. Mag. 38(6): 128-133.

Fliege, N. J. (1995). Multirate Digital Signal Processing, John Wiley \& Sons.

Furtado, M. B. J., Diniz, P. S. R., Netto, S. L. \& Saramäki, T. (2005). On the design of high-complexity cosine-modulated transmultiplexers based on the frequency-response masking approach, IEEE Trans. Circuits Syst. I 52(11): 2413-2426.

Ganesan, G. \& Li, Y. G. (2005). Cooperative spectrum sensing in cognitive radio networks, Proc. IEEE Int. Dynamic Spectrum Access Networks, pp. 137-143.

Göckler, H. G. \& Abdulazim, M. N. (2005). Joint oversampling FDM demultiplexing and perfectly reconstructing SBC filter bank for two channels, Proc. Eur. Signal Processing Conf., Antalya, Turkey.

Göckler, H. G. \& Abdulazim, M. N. (2007). Tree-structured MIMO FIR filter banks for flexible frequency reallocation, Proc. Int. Symp. Image Signal Processing Anal., Istanbul, Turkey.

Haykin, S. (2005). Cognitive radio: Brain-empowered wireless communications, IEEE J. Select. Areas Commun. 23(2): 201-220.

Heller, P. N. ., Karp, T. \& Nguyen, T. Q. (1999). A general formulation of modulated filter banks, IEEE Trans. Signal Processing 47(4): 986-1002.

Hentschel, T. \& Fettweis, G. (2000). Sample rate conversion for software radio, IEEE Commun. Mag. 38(8): 142-150.

Ihalainen, T., Stitz, T. H., Rinne, M. \& Renfors, M. (2007). Channel equalization in filter bank based multicarrier modulation for wireless communications, EURASIP J. Appl. Signal Processing 2007, Article ID 49389.

Johansson, H. \& Hermanowicz, E. (2006). Adjustable fractional-delay filters utilizing the Farrow structure and multirate techniques, Proc. Int. Workshop Spectral Methods Multirate Signal Processing, Florence, Italy.

Johansson, H. \& Löwenborg, P. (2003). On the design of adjustable fractional delay FIR filters, IEEE Trans. Circuits Syst. II 50(4): 164-169.

Johansson, H. \& Löwenborg, P. (2005). Flexible frequency band reallocation network based on variable oversampled complex-modulated filter banks, Proc. IEEE Int. Conf. Acoust. Speech Signal Processing, Philadelphia, USA. 
Johansson, H. \& Löwenborg, P. (2007). Flexible frequency-band reallocation networks using variable oversampled complex-modulated filter banks, EURASIP J. Adv. Signal Processing 2007, Article ID 63714.

Jondral, F. K. (2005). Software defined radio: Basics and evolution to cognitive radio, EURASIP J. Wireless Commun. Networking .

Jondral, F. K. (2007). Cognitive radio: A communications engineering view, IEEE Wireless Commun. Mag. 14(4): 28-33.

Karp, T. \& Fliege, N. J. (1999). Modified DFT filter banks with perfect reconstruction, IEEE Trans. Circuits Syst. II 46(11): 1404-1414.

Kovačević, J. \& Vetterli, M. (1993). Perfect reconstruction filter banks with rational sampling factors, IEEE Trans. Signal Processing 41(6): 2047-2066.

Leaves, P., Moessner, K., Tafazolli, R., Grandblaise, D., Bourse, D., Tonjes, R. \& Breveglieri, M. (2004). Dynamic spectrum allocation in composite reconfigurable wireless networks, IEEE Commun. Mag. 42(5): 72-81.

Li, J., Nguyen, T. Q. \& Tantaratana, S. (1997). A simple design method for near-perfect-reconstruction nonuniform filter banks, IEEE Trans. Signal Processing 45(8): 2105-2109.

Lin, Y.-P. \& Phoong, S.-M. (2001). ISI-free FIR filterbank transceivers for frequency-selective channels, IEEE Trans. Signal Processing 49(11): 2648-2658.

Lippolis, G., Simone, L., Comparing, M. C., Gelfusa, D., Piloni, V. \& Novello, R. (2004). Overview on band-pass sampling approaches for on-board processing, Proc. IEEE Int. Symp. Signal Processing Inf. Theory, pp. 543-548.

Lucente, M., Re, E., Rossi, T., Sanctis, M. D., Stallo, C., Cianca, E. \& Ruggieri, M. (2008). Future perspectives for the new European data relay system, Proc. IEEE Aerospace Conf., pp. 1-7.

Martin-Martin, P., Bregovic, R., Martin-Marcos, A., Cruz-Roldan, F. \& Saramäki, T. (2008). A generalized window approach for designing transmultiplexers, IEEE Trans. Circuits Syst. I 55(9): 2696-2706.

Martin-Martin, P., Cruz-Roldan, F. \& Saramäki, T. (2005). Optimized transmultiplexers for multirate systems, Proc. IEEE Int. Symp. Circuits Syst., Vol. 2, Kobe, Japan, pp. 1106-1109.

Mirabbasi, S. \& Martin, K. (2003). Overlapped complex-modulated transmultiplexer filters with simplified design and superior stopbands, IEEE Trans. Circuits Syst. II 50(5): 1170-1183.

Mitra, S. K. (2006). Digital Signal Processing: A Computer Based Approach, McGraw-Hill.

Nguyen, T., Hant, J., Taggart, D., Tsang, C.-S., Johnson, D. M. \& Chuang, J.-C. (2002). Design concept and methodology for the future advanced wideband satellite system, Proc. IEEE Military Commun. Conf., Vol. 1, USA, pp. 189-194.

Princen, J. (1994). The design of nonuniform modulated filter banks, Proc. IEEE Int. Symp. Time-Frequency Time-Scale Anal., pp. 112-115.

Pun, C. K. S., Wu, Y. C., Chan, S. C. \& Ho, K. L. (2003). On the design and efficient implementation of the Farrow structure, IEEE Signal Processing Lett. 10(7): 189-192.

Ramacher, U. (2007). Software-defined radio prospects for multistandard mobile phones, IEEE Computer 40(10): 62-69.

Re, E. D. \& Pierucci, L. (2002). Next-generation mobile satellite networks, IEEE Commun. Mag. 40(9): 150-159. 
Rosenbaum, L., Johansson, H. \& Löwenborg, P. (2006). Oversampled complex-modulated causal IIR filter banks for flexible frequency-band reallocation networks, Proc. Eur. Signal Processing Conf., Florence, Italy.

Saramäki, T. \& Bregović, R. (2001a). An efficient approach for designing nearly perfect-reconstruction cosine-modulated and modified DFT filter banks, Proc. IEEE Int. Conf. Acoust. Speech Signal Processing.

Saramäki, T. \& Bregovic, R. (2001b). An efficient approach for designing nearly perfect-reconstruction cosine-modulated and modified DFT filter banks, Proc. IEEE Int. Conf. Acoust. Speech Signal Processing, Vol. 6, pp. 3617-3620.

Sherman, M., Mody, A. N., Martinez, R., Rodriguez, C. \& Reddy, R. (2008). IEEE standards supporting cognitive radio and networks, dynamic spectrum access, and coexistence, IEEE Commun. Mag. 46(7): 72-79.

Tseng, C. C. (2002). Design of variable fractional delay FIR filter using differentiator bank, Proc. IEEE Int. Symp. Circuits Syst., Vol. 4, pp. 421-424.

Vaidyanathan, P. P. (1993). Multirate Systems and Filter Banks, Prentice-Hall, Englewood Cliffs, NJ.

Vaidyanathan, P. P. \& Vrcelj, B. (2004). Transmultiplexers as precoders in modern digital communications: A tutorial review, Proc. IEEE Int. Symp. Circuits Syst., Vol. 5, pp. $405-412$.

Vesma, J. \& Saramäki, T. (1996). Interpolation filters with arbitrary frequency response for all-digital receivers, Proc. IEEE Int. Symp. Circuits Syst.

Vesma, J. \& Saramäki, T. (1997). Optimization and efficient implementation of FIR filters with adjustable fractional delay, Proc. IEEE Int. Symp. Circuits Syst., Vol. IV, Hong Kong, pp. 2256-2259.

Vesma, J. \& Saramäki, T. (2000). Design and properties of polynomial-based fractional delay filters, Proc. IEEE Int. Symp. Circuits Syst., Geneva, Switzerland.

Viholainen, A., Alhava, J. \& Renfors, M. (2006). Efficient implementation of complex modulated filter banks using cosine and sine modulated filter banks, EURASIP J. Appl. Signal Processing 2006, Article ID 58564.

Viholainen, A., Saramäki, T. \& Renfors, M. (1999). Nearly perfect-reconstruction cosine-modulated filter banks for VDSL modems, Proc. IEEE Int. Conf. Electron. Circuits Syst., pp. 373-376.

Wittig, M. (2000). Satellite on-board processing for multimedia applications, IEEE Commun. Mag. 38(6): 134-140.

Xie, X. M., Chan, S. C. \& Yuk, T. I. (2005). Design of perfect-reconstruction nonuniform recombination filter banks with flexible rational sampling factors, IEEE Trans. Circuits Syst. I 52(9): 1965-1981.

Xie, X. M., Chen, X. Y. \& Sho, G. M. (2005). A simple design method of linear-phase nonuniform filter banks with integer decimation factors, Proc. IEEE Int. Midwest Symp. Circuits Syst., pp. 7-10.

Yuan, Y., Bahl, P., Chandra, R., Chou, P. A., Ferrell, J. I., Moscibroda, T., Narlanka, S. \& Wu, Y. (2007). KNOWS: Cognitive radio networks over white spaces, Proc. IEEE Int. Dynamic Spectrum Access Networks, pp. 416-427.

Yuan, Y., Bahl, P., Chandra, R., Moscibroda, T., Narlanka, S. \& Wu, Y. (2007). Allocating dynamic time-spectrum blocks in cognitive radio networks, Proc. ACM Int. Symp. Mobile Ad Hoc Networking Comput. 
Zhao, Q. \& Sadler, B. M. (2007). A survey of dynamic spectrum access, IEEE Signal Processing Mag. 24(3): 79-89.

Zhao, Q. \& Swami, A. (2007). A survey of dynamic spectrum access: Signal processing and networking perspectives, Proc. IEEE Int. Conf. Acoust. Speech Signal Processing, Vol. 4, pp. 1349-1352. 


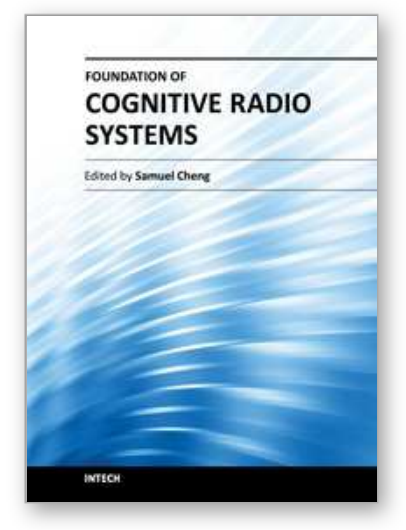

\author{
Foundation of Cognitive Radio Systems \\ Edited by Prof. Samuel Cheng
}

ISBN 978-953-51-0268-7

Hard cover, 298 pages

Publisher InTech

Published online 16, March, 2012

Published in print edition March, 2012

The fast user growth in wireless communications has created significant demands for new wireless services in both the licensed and unlicensed frequency spectra. Since many spectra are not fully utilized most of the time, cognitive radio, as a form of spectrum reuse, can be an effective means to significantly boost communications resources. Since its introduction in late last century, cognitive radio has attracted wide attention from academics to industry. Despite the efforts from the research community, there are still many issues of applying it in practice. This books is an attempt to cover some of the open issues across the area and introduce some insight to many of the problems. It contains thirteen chapters written by experts across the globe covering topics including spectrum sensing fundamental, cooperative sensing, spectrum management, and interaction among users.

\title{
How to reference
}

In order to correctly reference this scholarly work, feel free to copy and paste the following:

Amir Eghbali and Håkan Johansson (2012). Reconfigurable Multirate Systems in Cognitive Radios, Foundation of Cognitive Radio Systems, Prof. Samuel Cheng (Ed.), ISBN: 978-953-51-0268-7, InTech, Available from: http://www.intechopen.com/books/foundation-of-cognitive-radio-systems/reconfigurable-multirate-systems-incognitive-radios

\section{INTECH}

open science | open minds

\author{
InTech Europe \\ University Campus STeP Ri \\ Slavka Krautzeka 83/A \\ 51000 Rijeka, Croatia \\ Phone: +385 (51) 770447 \\ Fax: +385 (51) 686166 \\ www.intechopen.com
}

\author{
InTech China \\ Unit 405, Office Block, Hotel Equatorial Shanghai \\ No.65, Yan An Road (West), Shanghai, 200040, China \\ 中国上海市延安西路65号上海国际贵都大饭店办公楼405单元 \\ Phone: +86-21-62489820 \\ Fax: +86-21-62489821
}


(C) 2012 The Author(s). Licensee IntechOpen. This is an open access article distributed under the terms of the Creative Commons Attribution 3.0 License, which permits unrestricted use, distribution, and reproduction in any medium, provided the original work is properly cited. 